\title{
Isolated breast metastasis mimicking as second primary cancer - a case report
}

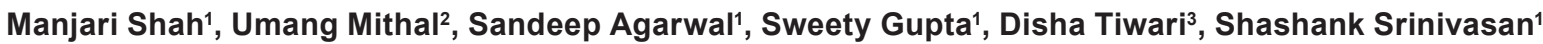 \\ Asheesh Jain ${ }^{4}$, Ritu Chandra ${ }^{1}$ \\ ${ }^{\prime}$ Department of Radiation Oncology, Max Hospital, Vaishali 201012, Delhi NCR, India. \\ ${ }^{2}$ Department of Surgical Oncology, Max Hospital, Vaishali 201012, Delhi NCR, India. \\ ${ }^{3}$ Department of Radiation oncology, King George Medical University, Lucknow 226003, Uttar Pradesh, India. \\ ${ }^{4}$ Consulting Histopathologist, Asheesh Pathology Lab, Uttar Pradesh 250002, India.
}

Correspondence to: Dr. Manjari Shah, Department of Radiation Oncology, Max Hospital, Vaishali 201012, Ghaziabad, Uttar Pradesh, India. E-mail: manjarishah29@gmail.com

How to cite this article: Shah M, Mithal U, Agarwal S, Gupta S, Tiwari D, Srinivasan S, Jain A, Chandra R. Isolated breast metastasis mimicking as second primary cancer - a case report. J Cancer Metastasis Treat 2016;2:391-5.

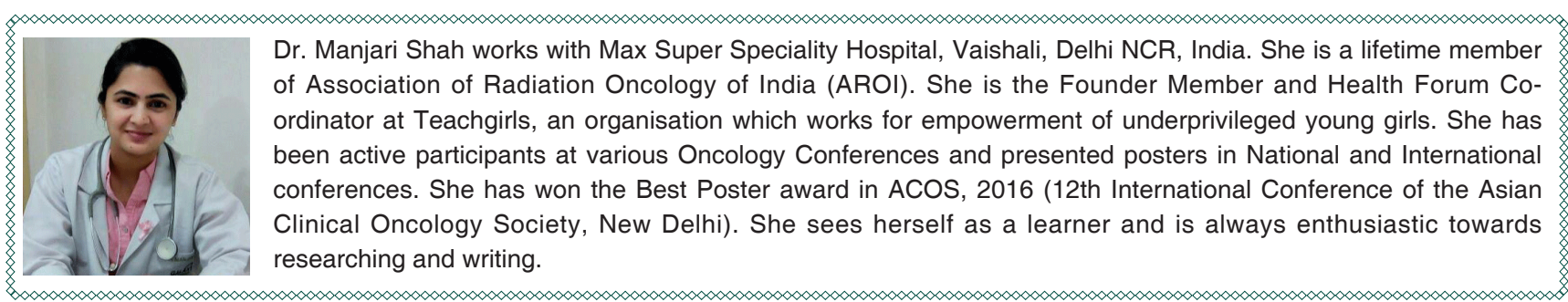

Article history:

Received: 03-05-2016

Accepted: 23-07-2016

Published: 30-09-2016

Key words:

Isolated breast metastasis,

second primary carcinoma of

breast,

carcinoma anorectum

\section{ABSTRACT}

Primary carcinoma of breast is common but breast is a rare site of metastasis and metastases from extramammary sites are even rarer. Metastasis to breast from rectal carcinoma is very unusual and till now 19 cases of breast secondaries from colorectal carcinoma have been reported in literature which include 14 cases where the primary site was colon and remaining 5 were from the rectum. Here the authors report a case of adenocarcinoma anorectum who had completed treatment and after 4 months developed a lump in her left breast which was metastatic. Metastatic lesions of breast are usually part of a widely disseminated disease but this case presented as a solitary breast metastasis which mimicked as second primary cancer of the breast.

\section{INTRODUCTION}

Breast is an unusual site of metastasis. ${ }^{[1]}$ Contralateral breast is the most common site from which breast metastases are seen, followed by extramammary sites, viz. leukemia, melanoma, lymphoma, ovary, lung and stomach cancer. ${ }^{[2,3]}$ Breast metastasis from extra mammary tumor is rare and accounts for 


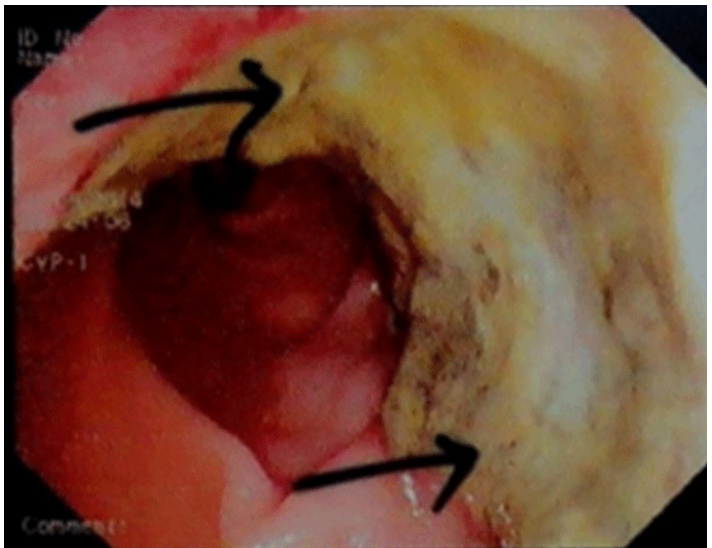

Figure 1: Colonoscopy reported circumferential ulcerative growth in distal rectum and anal canal as the arrows indicated

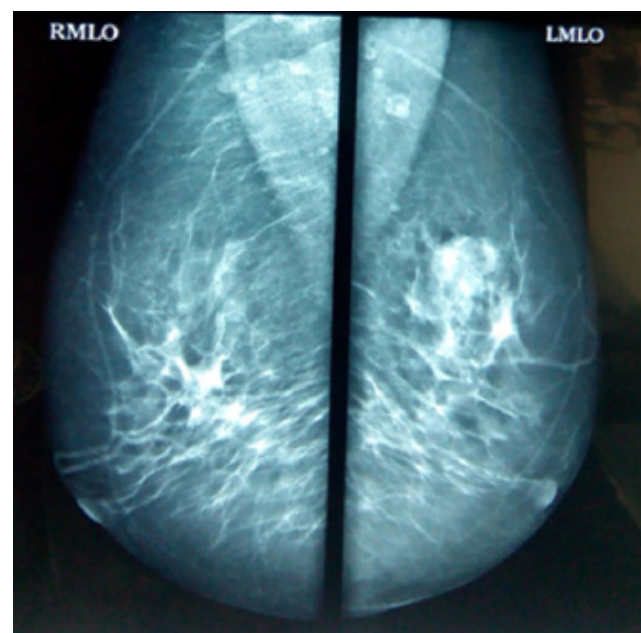

Figure 3: Digital mammography of bilateral breast showing oval hyperdense mass lesion with lobulated margins in upper outer quadrant of left breast

approximately $1.3 \%$ to $6.6 \%$ of all malignant tumors of breast. ${ }^{[4]}$ Metastasis from the colon to the breast were first reported by McIntosh et al. ${ }^{[5]}$ and from the rectum by Lal et al. ${ }^{[6]}$ in 1999. It is important to differentiate metastatic disease to the breast from primary breast carcinoma because the management differs in both the scenarios.

\section{CASE REPORT}

A 49-year-old female presented to oncology out patient department with complaints of bleeding per rectum and alteration of bowel habit since 1 month. The patient was will built and had Eastern Cooperative Oncology Group performance score of 1 . General physical examination was unremarkable. Per-rectal examination revealed ulcero-proliferative growth involving posterior wall of anal canal was palpable at $4 \mathrm{~cm}$ from the anal verge. Colonoscopy was done which reported circumferential ulcerative growth in distal rectum and anal canal [Figure 1]. Contrast enhanced computed tomography
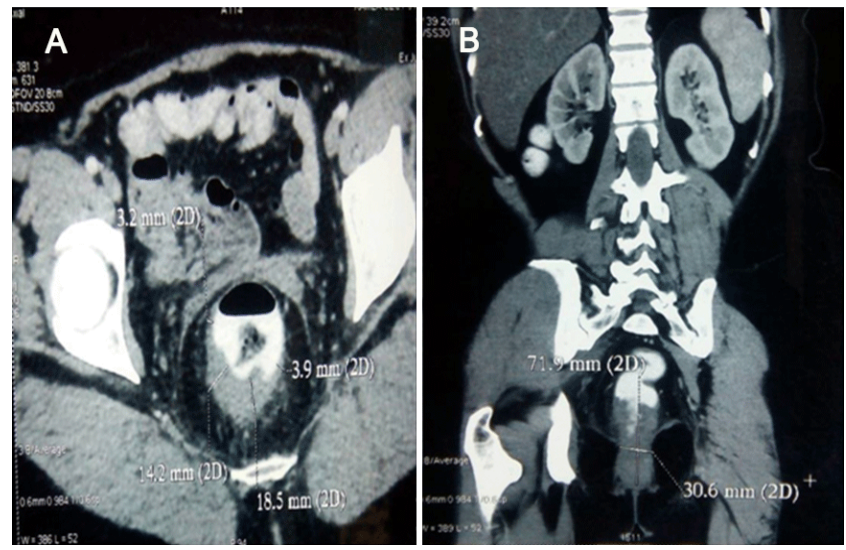

Figure 2: (A) Computed tomography scan of abdomen in axia section showing semi-circumferential mass lesion in anorectal region predominantly involving posterior wall; (B) computed tomography scan of abdomen in coronal section showing $71.9 \mathrm{~mm}$ mass lesion in anorectal region

Table 1: IHC markers results in our patient

\begin{tabular}{lc}
\hline IHC markers & Results \\
\hline CK7 & Negative \\
CK20 & Positive in majority of tumor cells \\
mCEA & Positive in majority of tumor cells \\
ER & Negative, normal breast is positive \\
GCDFP-15 & Negative \\
MUC-2 & Positive in many tumor cells \\
CDX-2 & Positive in many tumor cells \\
Ki-67 & $30 \%$ \\
\hline
\end{tabular}

IHC: immunohistochemistry; $\mathrm{CK}$ : cytokeratin; mCEA: carcinoembryonic antigen; ER: estrogen receptor; GCDFP-15: gross cystic disease fluid protein; MUC-2: mucin-2; CDX-2: Caudal type homeobox-2

scan of the whole abdomen was done which showed semi-circumferential mass lesion (length $71.9 \mathrm{~mm}$; width $30.6 \mathrm{~mm}$; thickness of mass $3.2 \mathrm{~mm}$ to $18.5 \mathrm{~mm}$ ) in anorectal region predominantly involving posterior wall [Figure 2A and 2B]. All other baseline investigations including a complete hemogram, kidney function tests, liver function tests, and chest $\mathrm{X}$-ray were within normal limits. Biopsy from anorectal mass revealed signet ring adenocarcinoma. She underwent pre-operative external beam radiotherapy $50.4 \mathrm{~Gy}$ in 28 fractions with concomitant 5-fluorouracil and leucovorin based chemotherapy followed by radical surgery (abdominoperineal resection with permanent colostomy) and then adjuvant 5-fluorouracil and leucovorin based chemotherapy. Patient was disease free for 4 months after completion of treatment, and 4 months after completion of treatment, she noticed a lump in her left breast. On clinical examination a lump was palpable approximately $2 \mathrm{~cm} \times 2 \mathrm{~cm}$ size in the upper outer quadrantofleftbreastwithnoaxillaryand supraclavicular lymphadenopathy. Digital mammography of bilateral breast was done which revealed oval hyperdense mass lesion with lobulated margins in upper outer quadrant of left breast [Figure 3]. She then underwent 


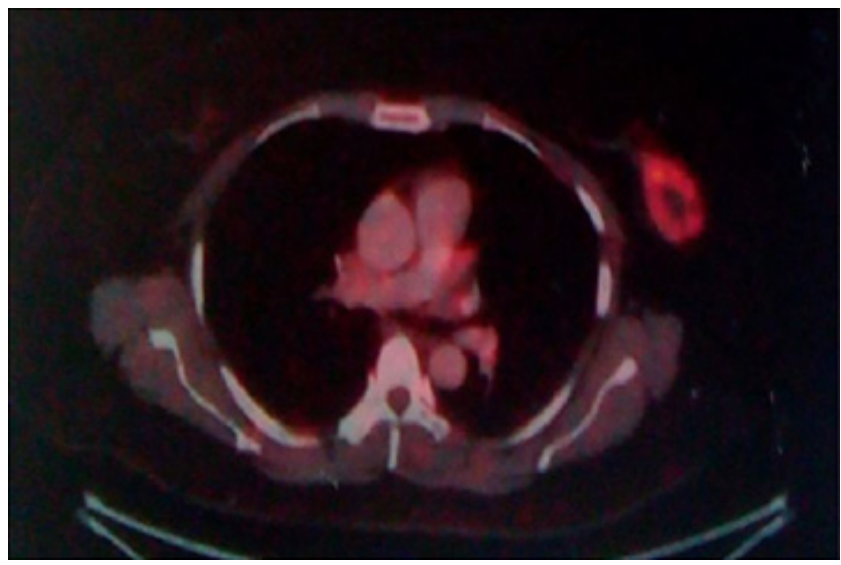

Figure 4: FDG-PET scan showed FDG avid soft tissue density lesion (size $4.2 \mathrm{~cm} \times 2.8 \mathrm{~cm} \mathrm{SUV}_{\max }$ 13.2) in left breast. FDGPET: fluorodeoxyglucose-positron emission tomography; SUV: standardized uptake value

a whole body 18-fluorodeoxyglucose (18-FDG) positron emission tomography scan which showed FDG avid soft tissue density lesion of size $4.2 \mathrm{~cm}$ $\times 2.8 \mathrm{~cm}$ with standardized uptake value (SUV) 13.2 in left breast [Figure 4] and hypermetabolic right inguinal lymph node SUV max 5.1 [Figure 5] with no other hypermetabolic focus elsewhere in body. Fine needle aspiration cytology (FNAC) from left breast lump showed single population of atypical epithelial cells suggestive of adenocarcinoma. FNAC from right inguinal node was also done which reported metastasis from adenocarcinoma. Her carcinoembryonic antigen (CEA) and carbohydrate antigen-15.3 was done which was $26.8 \mathrm{ng} / \mathrm{mL}$ (Normal 0-4 ng/mL) and $17.2 \mathrm{u} / \mathrm{mL}$ (Normal 0-35 u/mL) respectively. In view of isolated breast lesion it was considered as second primary of the breast and the patient was taken up for left modified radical mastectomy. Right iliac and inguinal node dissection was also performed for regional lymph node recurrence from carcinoma anorectum. Postoperative histopathology from left modified radical mastectomy specimen showed mucin secreting signet ring adenocarcinoma with lymphovascular emboli and lymphocytic infiltration. Nine out of 16 dissected left axillary lymph nodes showed metastasis of signet ring adenocarcinoma. Six out of 8 right inguinal lymph nodes and 2 out of 4 right iliac lymph nodes showed metastasis from anorectal carcinoma. Immunohistochemistry (IHC) was performed to ascertain whether the lesion was a primary carcinoma of the breast or metastasis from anorectal carcinoma. Result of IHC markers was as shown in Table 1 and Figure 6. IHC combined with morphology favored signet ring cell metastatic carcinoma to breast.

\section{DISCUSSION}

Breast metastases from colon cancer are very rare and

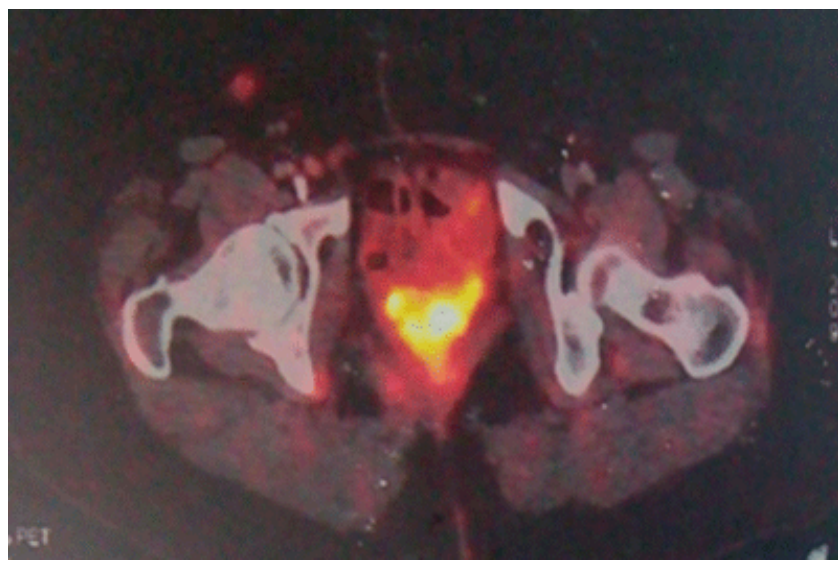

Figure 5: FDG avid hypermetabolic right inguinal lymph node SUV $_{\max }-5.1$. FDG: fluorodeoxyglucose; SUV: standardized uptake value

they are usually associated with poor prognosis, due to disseminated disease. ${ }^{[7]}$ It is of utmost importance to distinguish metastatic carcinoma to the breast from a primary breast carcinoma. ${ }^{[8]}$ Metastatic spread from anorectal cancer occurs both by lymphatic and hematogenous routes. Owing to the venous drainage into the portal system from the superior hemorrhoidal vein, the liver is the most common site of distant metastasis. Systemic drainage into the inferior vena cava from the inferior hemorroidal plexus may lead to metastatic involvement of the lung and bone. Metastases to the breast from anorectal carcinoma without involvement of any of these organs is a rare phenomenon. Schaekelford et al. ${ }^{[8]}$ reviewed 19 cases of colorectal carcinoma metastasizing to the breast and reported a majority of cases with metastases to the left breast $55 \%$, with the right breast $30 \%$ and 3 cases with bilateral breast metastasis. In our case, patient had left breast metastasis similar to the observation by Schaekelford et al. ${ }^{[9]}$ The most common site is the upper outer quadrant of the breast. They can occur as synchronous lesions or may follow the primary by months to years. Metastatic breast lesions are typically mobile, well demarcated, firm, rapidly growing, discrete masses and may be confused with benign breast disease due to their often well-circumscribed nature. Rarely these lesions may be multiple or bilateral. The interpretation is difficult in some cases so a history of previous malignancy is important for the radiologist in order to evaluate these breast lesions. ${ }^{[10,11]}$ Other features suggestive of metastasis to breast are location of the lump in either fat or subcutaneous tissue, lack of micro-calcification in mammogram and lack of in situ disease on histopathological examination. ${ }^{[12,13]}$ The correct diagnosis is therefore crucial in these patients so as to decide the further management of these patients. Histopathology for metastatic lesion may be invasive adenocarcinoma, often with mucinous or signet-ring cell features, but unlike primary lesion of the 


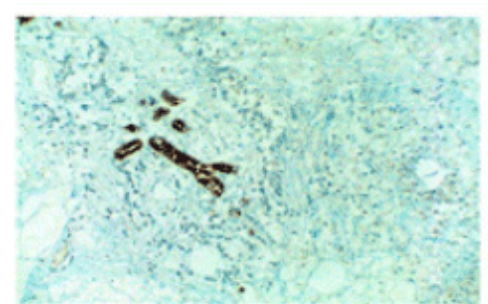

CK7 negative

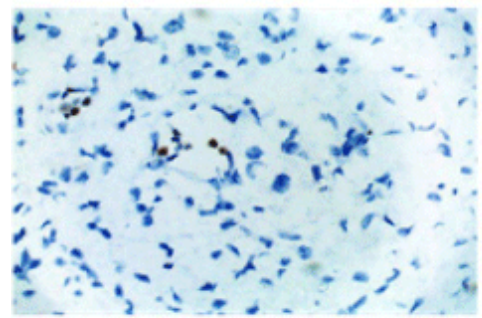

ER negative

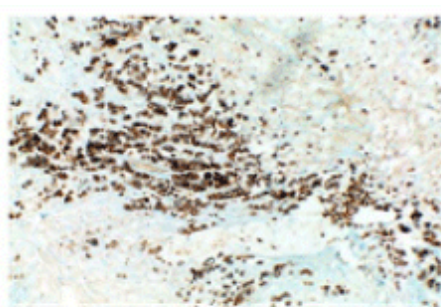

CK20 positive

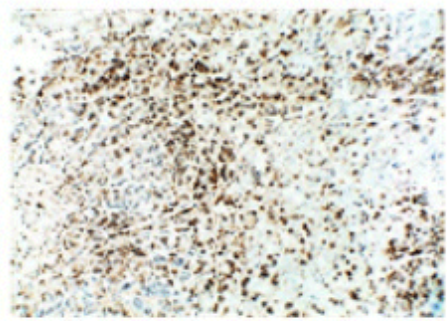

MUC2 positive

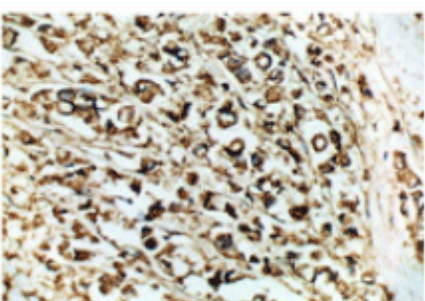

mCEA positive

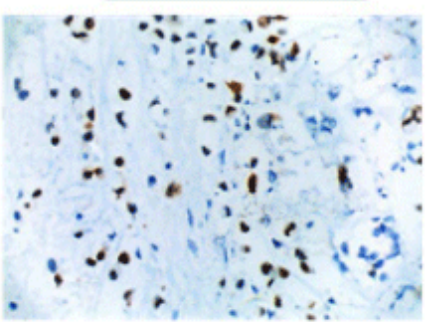

CDX2 positive

Figure 6: Immunohistochemistry markers results in our patient. CK: cytokeratin; mCEA: carcinoembryonic antigen; ER: estrogen receptor; MUC2: mucin2; CDX2: Caudal type homeobox2

breast they lack an in situ component. Lymphovascular space invasion may be prominent. This type of unusual histopathology in breast with previous history of malignancy are suggestive of metastasis. But the final diagnosis is established after studying the cytokeratin pattern. IHC when performed, tends to be positive for colorectal markers like caudal type homeobox-2 (CDX2), cytokeratin (CK20), and CEA, and negative for breast markers $\mathrm{CK} 7$, estrogen receptor, progesterone receptor, human epidermal growth factor receptor-2, and gross cystic disease fluid protein-15. ${ }^{[14,15]}$ Expression of CK7 and CK20 is considered to be most helpful in identifying the origin of adenocarcinomas.

Most importantly, the great majority of primary breast tumors are CK7-positive and CK20-negative, while colorectal carcinomas are usually CK7-negative and CK20-positive. ${ }^{[16,17]} \mathrm{IHC}$ markers used in our case were consistent with these findings as shown in Table 1. The strong nuclear positivity with CDX-2 is highly sensitive and specific for colonic cancers. ${ }^{[18]}$ In addition, estrogen and progesterone receptors are usually negative in metastatic breast cancers. A patchy reaction for CK5/6 and comedo like necrosis can mimic ductal carcinoma in situ disease. Histological features such as epithelial stratification, high nuclear atypia, significant mitotic activity, and positive reactions for CK20 and CDX-2 can help to overcome this difficulty. Metastatic carcinomas in the breast are associated with a poor prognosis with a survival rate of less than 12 months from the time of breast tumor diagnosis. ${ }^{[16,19,20]}$

Metastatic disease in the breast is a marker for disseminated metastatic spread, and therefore indicates a poor prognosis. Metastases to the breast are rare in themselves, and such metastasis occurring secondary to a previous anorectal carcinoma makes this case very unusual. The liver, lungs and bone are the usual sites of spread from colorectal cancers. Breast metastases with sparing of these organs is unlikely but possible. Our patient presented with an isolated breast lump and without any other complaints. She was managed considering the lesion to be second primary cancer of the breast but post operative histopathology with IHC showed it to be metastases. On the basis of histopathology showing adenocarcinoma and history of previous malignancy alone, the diagnosis of lesion being metastasis to breast should not be arrived upon and in such patients the importance of IHC to exclude the diagnosis of primary breast lesion cannot be undermined.

\section{Financial support and sponsorship Nil.}

\section{Conflicts of interest}

There are no conflicts of interest.

\section{Patient consent \\ Obtained.}

\section{Ethics approval}

Ethics approval was obtained prior to the commencement of the study.

\section{REFERENCES}

1. Hajdu SI, Urban JA. Cancers metastatic to the breast. Cancer 
1972;29:1691-6.

2. Moore DH, Wilson DK, Hurteau JA, Look KY, Stehman FB, Sutton GP. Gynecologic cancers metastatic to the breast. $J$ Am Coll Surg 1998;187:178-81.

3. Chaignaud B, Hall TJ, Powers C, Subramony C, Scott-Conner CE. Diagnosis and natural history of extramammary tumors metastatic to the breast. J Am Coll Surg 1994;179:49-53.

4. Bassi F, Gatti G, Mauri E, Ballardini B, De Pas T, Luini A. Breast metastases from cutaneous malignant melanoma. Breast 2004;13:533-5.

5. McIntosh IH, Hooper AA, Millis RR. Greening WP. Metastatic carcinoma within the breast. Clin Oncology 1976;2:393-401.

6. Lal RL, Joffe JK. Rectal carcinoma metastatis to the breast. Clin Oncol (R Coll Radiol) 1999;11:422-3.

7. Barthelmes L, Simpson JS, Douglas-Jones AG, Sweetland HM. Metastasis of primary colon cancer to the breast - leave well alone. Breast Care (Basel) 2010;5:23-5.

8. Shackelford RE, Allam-Nandyala P, Bui MM, Kiluk JV, Esposito NN. Primary colorectal adenocarcinoma metastatic to the breast: case report and review of nineteen cases. Case Rep Med 2011;2011:738413.

9. Chaignaud B, Hall TJ, Powers C, Subramony C, Scott-Conner CE. Diagnosis and natural history of extramammary tumors metastatic to the breast. J Am Coll Surg 1994;179:49-53.

10. Deeley TJ. Secondary deposits in the breast. Br J Cancer 1965;19:738-43.

11. Paulus DD, Lidsith HI. Metastasis to the breast. Radiolo Clini North Am 1982;20:561-8.

12. Harvey EB and Brinton IA. Second cancer following cancer of breast in connecticut 1935-82. Natl Cancer Inst Monogr 1985;68:99-112.
13. Lewison EF, Neto AS. Bilateral breast cancer at johns hopkins hospital. A discussion of dilemma of contralateral breast cancer. Cancer 1971;28:1297-301.

14. Chinyama CN, Davies JD. Mammary mucinous lesions: congeners, prevalence and important pathological associations. Histopathology 1996;29:533-9.

15. Lagendijk JH, Mullink H, van Diest PJ, Meijer GA, Meijer CJ. Immunohistochemical differentiation between primary adenocarcinomas of the ovary and ovarian metastases of colonic and breast origin. Comparison between stadistical and intuitive approach. J Clin Pathol 1999;52:283-90.

16. Ahmad A, Baiden-Amissah K, Oyegade A, Absar M, Swainson K Titi S. Primary sigmoid adenocarcinoma metastasis to the breast in a 28 year old female : a case study and review of literature. Korean $J$ Pathol 2014;48:58-61

17. Noh KT, Oh B, Sung SH, Lee RA, Chung SS, Moon BI, Kim KH Metastasis to the breast from colonic adenocarcinoma. J Korean Surg Soc 2011;81 Suppl 1:S43-6.

18. Cabibi D, Cipolla C, Valerio MR, Ingrao S. Metastatic seeding of colon adenocarcinoma manifesting as synchronous breast and chest wall localization: report of a case. Surg Today 2011;41:242-6.

19. Ho YY, Lee WK. Metastasis to the breast from an adenocarcinoma of the colon. J Clin Ultrasound 2009;37:239-41.

20. Perin T, Canzonieri V, Memeo L, Massarut S. Breast metastasis of primary colon cancer with micrometastasis in the axillary sentinel node: a metastasis that metastasized? Diagn Pathol 2011;6:45. 